\title{
ASYMPTOTIC BEHAVIOUR OF RAMANUJAN'S TENTH ORDER MOCK THETA FUNCTIONS
}

\author{
BHASKAR SRIVASTAVA
}

\section{Introduction}

In his last letter to Hardy, Ramanujan [3, p. 474] described a set of functions defined by Eulerian series inside the unit circle, having asymptotic representations at their essential singularities of the same order of precision as those known for the $\theta$-functions, and he called them mock theta functions. Ramanujan also explained what he meant by mock theta function [1, p. 63]. It is a function $f(q)$ defined by a $q$-series which converges for $|q|<1$ and satisfies the following two conditions:

(0) For every root of unity $\zeta$, there is a $\theta$-function $\theta_{\zeta}(q)$ such that the difference $f(q)-\theta_{\zeta}(q)$ is bounded as $q \rightarrow \zeta$ radially.

(1) There is no single $\theta$-function which works for all $\zeta$ i.e., for every $\theta$ function $\theta(q)$ there is some root of unity $\zeta$ for which $f(q)-\theta(q)$ is unbounded as $q \rightarrow \zeta$ radially.

In $[4$, p. 9] Ramanujan defined four more mock theta functions which were called by Choi $[2$, p. 498$]$ tenth order mock theta functions. In this paper we will show that these four tenth order mock theta functions are bounded near certain roots of unity. We shall also show these functions satisfy condition (0). We will mainly follow the method used by Andrews and Hickerson [1, pp. 92-96] for sixth order mock theta functions and Watson [5, p. 292-295] for fifth order mock theta functions, in proving the results.

\section{Notation and definition}

The following $q$-notation is used: For $\left|q^{k}\right|<1$

$$
\begin{aligned}
\left(a ; q^{k}\right)_{n} & =(1-a)\left(1-a q^{k}\right) \cdots\left(1-a q^{k(n-1)}\right), \quad n \geq 1 \\
\left(a ; q^{k}\right)_{o} & =1, \\
(a)_{n} & =(a ; q)_{n}=(1-a)(1-a q) \cdots\left(1-a q^{n-1}\right) .
\end{aligned}
$$

2000 Mathematics Subject Classification: 33D.

Keywords and phrases: Mock theta functions, basic hypergeometric series.

Received July 24, 2001. 
The four tenth order mock theta functions as defined by Ramanujan [4, p. 9] are

$$
\begin{aligned}
\Phi(q) & :=\sum_{n=0}^{\infty} \frac{q^{n(n+1) / 2}}{\left(q ; q^{2}\right)_{n+1}} \\
\Psi(q) & :=\sum_{n=0}^{\infty} \frac{q^{(n+1)(n+2) / 2}}{\left(q ; q^{2}\right)_{n+1}} \\
X(q) & :=\sum_{n=0}^{\infty} \frac{(-1)^{n} q^{n^{2}}}{(-q ; q)_{2 n}}
\end{aligned}
$$

and

$$
\chi(q):=\sum_{n=0}^{\infty} \frac{(-1)^{n} q^{(n+1)^{2}}}{(-q ; q)_{2 n+1}}
$$

\section{The behaviour of the mock theta functions in the neighbourhood of the unit circle}

According to Ramanujan [5, p. 292] the characteristic property of a mock theta function is that corresponding to each "rational point" $q=e^{\pi i(h / k)},(h$ and $k$ integers and $(h, k)=1)$ of the unit circle $|q|=1$, there exist a $\theta$-function of $q$ whose difference from the mock theta function is bounded when $q \rightarrow$ this rational point along a radius of the circle.

The rational numbers $h / k$, expressed in their lowest terms can be put into three categories:

(i) $h$ even and $k$ odd

(ii) $h$ and $k$ both odd

(iii) $h$ odd and $k$ even

We shall now prove the following theorems:

THeOREM 1. Let $q=\rho e^{\pi i(h / k)}, \rho$ is real and $0<\rho \leq 1$, and let $\rho \rightarrow 1$. Then for approach to $|q|=1$ along a radius of the first category, i.e., $h$ is even and $k$ is odd

$$
\Phi(q)=O(1) \quad \text { and } \quad \Psi(q)=O(1) .
$$

Proof. Let $q=\rho e^{\pi i(h / k)}, h$ is even, $k$ is odd, $(h, k)=1$.

Let

$$
\begin{aligned}
U_{n}(\rho) & =\frac{q^{n(n+1) / 2}}{\left(q ; q^{2}\right)_{n+1}} \\
& =\frac{\rho^{n(n+1) / 2} e^{\pi i(h / k) n(n+1) / 2}}{\prod_{p=0}^{n}\left(1-\rho^{2 p+1} e^{\pi i(h / k)(2 p+1)}\right)} .
\end{aligned}
$$


Then

$$
\left|U_{n+N}(\rho)\right|=\frac{\rho^{N n+N(N+1) / 2}}{\left|\prod_{p=0}^{N-1}\left(1-\rho^{2 n+3+2 p} e^{\pi i(h / k)(2 n+3+2 p)}\right)\right|}\left|U_{n}(\rho)\right| .
$$

We shall estimate the denominator.

Taking $R=\rho^{2 n+3}, R^{\prime}=\rho^{2 n+3+2 p}$ and $z=-e^{\pi i(h / k)(2 n+3+2 p)}$ in Lemma 5.2 of Andrews and Hickerson [1, p. 93], we have

$$
\begin{aligned}
\left|\prod_{p=0}^{N-1}\left(1-\rho^{2 n+3+2 p} e^{\pi i(h / k)(2 n+3+2 p)}\right)\right| & \geq \prod_{p=0}^{N-1} \rho^{p}\left|1-\rho^{2 n+3} e^{\pi i(h / k)(2 n+3+2 p)}\right| \\
& =\rho^{N(N-1) / 2}\left|\prod_{p=0}^{N-1}\left(1-\rho^{2 n+3} e^{\pi i(h / k)(2 n+3+2 p)}\right)\right| .
\end{aligned}
$$

As $p$ ranges from 0 to $N-1,1-\rho^{2 n+3} e^{\pi i(h / k)(2 n+3+2 p)}$ runs twice through the roots of the polynomial

$$
(x-1)^{N / 2}+(-1)^{N / 2} \rho^{N(2 n+3) / 2} .
$$

So the product of these roots is $1+\rho^{N(2 n+3) / 2}>1$

Hence

$$
\left|\prod_{p=0}^{N-1}\left(1-\rho^{2 n+3+2 p} e^{\pi i(h / k)(2 n+3+2 p)}\right)\right|>\rho^{N(N-1) / 2} .
$$

Hence for $0<\rho \leq 1$,

$$
\begin{aligned}
\left|U_{n+N}(\rho)\right| & \leq \rho^{n N+N}\left|U_{n}(\rho)\right| \\
& \leq \varepsilon\left|U_{n}(\rho)\right|, \quad 0<\varepsilon \leq 1 .
\end{aligned}
$$

Hence by Lemma 5.1 of Andrews and Hickerson [1, p. 93], $\sum_{n=0}^{\infty}\left|U_{n}(\rho)\right|$ is bounded for $0<\rho \leq 1$. Hence

Since

$$
\Phi(q)=\sum_{n=0}^{\infty}\left|U_{n}(\rho)\right|=O(1)
$$

$$
\Psi(q)=\sum_{n=0}^{\infty} q^{n+1}\left|U_{n}(\rho)\right|
$$

so

$$
\Psi(q)=O(1) .
$$


Theorem 2. Let $q=\rho e^{\pi i(h / k)}, \rho$ is real and $0<\rho \leq 1$ and let $\rho \rightarrow 1$. Then for approach to $|q|=1$ along a radius of the second category, i.e., $h$ and $k$ are both odd

$$
X(q)=O(1) \quad \text { and } \quad \chi(q)=O(1) .
$$

Proof. Let $q=\rho e^{\pi i(h / k)} h$ and $k$ be both odd, $(h, k)=1$.

Let

$$
\begin{aligned}
v_{n}(\rho) & =\frac{(-1)^{n} q^{n^{2}}}{(-q)_{2 n}} \\
& =\frac{(-1)^{n} \rho^{n^{2}} e^{\pi i(h / k) n^{2}}}{\prod_{p=0}^{2 n}\left(1+q^{p}\right)} .
\end{aligned}
$$

Then

$$
\left|v_{n+N}(\rho)\right|=\frac{\rho^{2 N n+N^{2}}}{\prod_{p=1}^{2 N}\left|\left(1+\rho^{2 n+p} e^{\pi i(h / k)(2 n+p)}\right)\right|}\left|v_{n}(\rho)\right| .
$$

We estimate the denominator.

Taking $R=\rho^{2 n+1}, R^{\prime}=\rho^{2 n+p}$ and $z=e^{\pi i(h / k)(2 n+p)}$ in Lemma 5.2 of Andrews and Hickerson [1, p. 93], we have

$$
\begin{aligned}
\prod_{p=1}^{2 N}\left|1+\rho^{2 n+p} e^{\pi i(h / k)(2 n+p)}\right| & \geq \prod_{p=1}^{2 N} \rho^{(p-1) / 2}\left|1+\rho^{2 n+1} e^{\pi i(h / k)(2 n+p)}\right| \\
& =\rho^{N^{2}-(N / 2)}\left|\prod_{p=1}^{2 N}\left(1+\rho^{2 n+1} e^{\pi i(h / k)(2 n+p)}\right)\right| \\
& =\rho^{N^{2}-(N / 2)}\left(1+\rho^{N(2 n+1)}\right)^{2} \\
& >\rho^{N^{2}-(N / 2)}
\end{aligned}
$$

since $1+\rho^{2 n+1} e^{\pi i(h / k)(2 n+p)}$ runs twice through the roots of

$$
(x-1)^{N}-\rho^{N(2 n+1)} .
$$

Hence

$$
\begin{aligned}
\left|v_{n+N}(\rho)\right| & \leq \rho^{2 N n+(N / 2)}\left|v_{n}(\rho)\right| \\
& \leq \varepsilon\left|v_{n}(\rho)\right|, \quad 0<\varepsilon \leq 1 .
\end{aligned}
$$

Hence by Lemma 5.1 of Andrews and Hickerson [1, p. 93] $\sum_{n=0}^{\infty}\left|v_{n}(\rho)\right|$ is bounded for $0<\rho \leq 1$. Hence 


$$
X(q)=\sum_{n=0}^{\infty} U_{n}(\rho)=O(1)
$$

Now

$$
\chi(q)=\sum_{n=0}^{\infty} \frac{q^{2 n+1}}{1+q^{2 n+1}} v_{n}(\rho)
$$

Now

$$
\begin{aligned}
\left|\frac{q^{2 n+1}}{1+q^{2 n+1}}\right| & =\frac{\rho^{2 n+1}}{\left|1+\rho^{2 n+1} e^{\pi i(h / k)(2 n+1)}\right|} \\
& \leq 1
\end{aligned}
$$

Hence

$$
\chi(q)=O(1)
$$

We shall now show that these functions satisfy condition $(0)$.

By Theorem 1, $\Phi(q)$ is bounded when $h$ is even and $k$ is odd and so if we take $\theta_{e^{\pi i(h / k)}}=0$, the condition $(0)$ is obviously satisfied. Same argument holds for $\Psi(q)$.

If $h$ and $k$ both are odd

$$
q^{2}=\rho^{2}\left(e^{\pi i(h / k)}\right)^{2}=\rho^{2} e^{\pi i(2 h / k)},
$$

So $2 h$ is even and $k$ is odd and by Theorem $2, X\left(q^{2}\right)$ and $\chi\left(q^{2}\right)$ will be bounded. So by the above argument both $X\left(q^{2}\right)$ and $\chi\left(q^{2}\right)$ satisfy condition $(0)$.

\section{REFERENCES}

[1] G. E. Andrews AND D. Hickerson, Ramanujan's "Lost" Notebook VII: The sixth order mock theta functions, Adv. Math., 89 (1991), 60-105.

[2 ] Y.-S. CHоI, Tenth order mock theta functions in Ramanujan's Lost Notebook, Invent. Math., 136 (1999), 497-569.

[3] L. A. Dragonette, Some asymptotic formulae for the mock theta series of Ramanujan, Trans. Amer. Math. Soc., 72 (1952), 474-500.

[4] S. Ramanujan, The Lost Notebook and Other Unpublished Papers, Narosa Publishing House, New Delhi, 1988.

[ 5 ] G. N. Watson, The mock theta functions (2), Proc. London Math. Soc., 42 (1936), $274-304$.

Department of Mathematics

LUCKNOW UNIVERSITY

LUCKNOW, INDIA 